\title{
Fundamental physics and the absence of sub-millisecond pulsars
}

\author{
B. Haskell ${ }^{1}$, J. L. Zdunik ${ }^{1}$, M. Fortin ${ }^{1}$, M. Bejger ${ }^{1,2}$, R. Wijnands ${ }^{3}$, and A. Patruno ${ }^{4}$ \\ 1 Nicolaus Copernicus Astronomical Center, Polish Academy of Sciences, Bartycka 18, 00-716 Warszawa, Poland \\ e-mail: bhaskell@camk.edu.pl \\ 2 APC, AstroParticule et Cosmologie, Université Paris Diderot, CNRS/IN2P3, CEA/Irfu, Observatoire de Paris, Sorbonne Paris Cité, \\ 75205 Paris Cedex 13, France \\ 3 Anton Pannekoek Institute for Astronomy, University of Amsterdam, Science Park 904, 1098 XH Amsterdam, The Netherlands \\ ${ }^{4}$ Leiden Observatory, Leiden University, PO Box 9513, 2300 RA Leiden, The Netherlands
}

Received 29 May 2018 / Accepted 21 September 2018

\begin{abstract}
Context. Rapidly rotating neutron stars are an ideal laboratory to test models of matter at high densities. In particular, the maximum rotation frequency of a neutron star depends on the equation of state and can be used to test models of the interior. However, observations of the spin distribution of rapidly rotating neutron stars show evidence for a lack of stars spinning at frequencies higher than $f \approx 700 \mathrm{~Hz}$, well below the predictions of theoretical equations of state. This has generally been taken as evidence of an additional spin-down torque operating in these systems, and it has been suggested that gravitational wave torques may be operating and be linked to a potentially observable signal. Aims. We aim to determine whether additional spin-down torques (possibly due to gravitational wave emission) are necessary, or if the observed limit of $f \approx 700 \mathrm{~Hz}$ could correspond to the Keplerian (mass-shedding) break-up frequency for the observed systems, and is simply a consequence of the currently unknown state of matter at high densities.

Methods. Given our ignorance with regard to the true equation of state of matter above nuclear saturation densities, we make a minimal physical assumption and only demand causality, that is, that the speed of sound in the interior of the neutron star should be lower than or equal to the speed of light $c$. We then connected our causally limited equation of state to a realistic microphysical crustal equation of state for densities below nuclear saturation density. This produced a limiting model that gave the lowest possible maximum frequency, which we compared to observational constraints on neutron star masses and frequencies. We also compared our findings with the constraints on the tidal deformability obtained in the observations of the GW170817 event.

Results. We rule out centrifugal breakup as the mechanism preventing pulsars from spinning faster than $f \approx 700 \mathrm{~Hz}$, as the lowest breakup frequency allowed by our causal equation of state is $f \approx 1200 \mathrm{~Hz}$. A low-frequency cutoff, around $f \approx 800 \mathrm{~Hz}$ could only be possible when we assume that these systems do not contain neutron stars with masses above $M \approx 2 M_{\odot}$. This would have to be due either to selection effects, or possibly to a phase transition in the interior of the neutron star that leads to softening at high densities and a collapse to either a black hole or a hybrid star above $M \approx 2 M_{\odot}$. Such a scenario would, however, require a somewhat unrealistically stiff equation of state for hadronic matter, in tension with recent constraints obtained from gravitational wave observations of a neutron star merger.
\end{abstract}

Key words. dense matter - stars: neutron - X-rays: binaries

\section{Introduction}

Neutron stars (NSs) are extraordinary cosmic laboratories, as they allow us to probe aspects of all fundamental interactions at extremes of density and gravity that are impossible to reproduce in terrestrial experiments. While current methods allow studying the physics of crustal layers below nuclear saturation density $\rho_{0}=2.7 \times 10^{14} \mathrm{~g} \mathrm{~cm}^{-3}$, the density in the core of an NS can exceed $\rho_{0}$ by an order of magnitude, entering a highly uncertain regime that is well beyond the possibilities of rigorous $\mathrm{ab}$ initio theoretical modelling (for a textbook introduction, see Haensel et al. 2007).

Constraints on theory have come from the observation of NSs with masses close to $2 M_{\odot}$ : PSR J1614-2230 (Demorest et al. 2010; Fonseca etal. 2016; Arzoumanian et al. 2018) and PSR J0348+0432 (Antoniadis etal. 2013), as well as from recent indications that PSR J2215+5135 may have a mass of $M \approx 2.3 M_{\odot}$ (Linares et al. 2018). These systems allow us to constrain several models for the equation of state (EOS) of dense matter by imposing that matter in the core must be stiff enough to allow for a maximum mass $M_{\max }>2 M_{\odot}$ (see e.g. Fortin et al
2016). More stringent constraints are expected in the near future, when data from NICER (Arzoumanian et al. 2014), and further in the future, from the ATHENA (Motch et al. 2013), mission will allow us to accurately determine pulse profiles and thus not only measure the mass, but also constrain the radius of several NSs.

While simultaneous measurements of mass and radius would allow for strong constraints on the EOS, it is important to keep in mind that many NSs rotate rapidly, with frequencies $f$ of up to $f \approx 700 \mathrm{~Hz}$ (Papitto et al. 2014; Hessels et al. 2006; Haensel et al. 2016; Patruno et al. 2017a; Bassa et al. 2017). Rapid rotation leads to increased centrifugal support and allows for the existence of supramassive NSs (objects with a mass higher than the maximum mass of the static configuration). This not only alters the mass-radius curve for a given EOS and must be kept in mind when analysing observational data, such as will be provided by NICER (Sieniawska et al. 2018), but may lead to a spin-down-induced collapse, which has been suggested as a possible engine for energetic phenomena such as fast radio bursts (Falcke \& Rezzolla 2014; Ravi \& Lasky 2014), or the 
short-lived X-ray afterglows observed in some short gamma-ray bursts (Dai \& Lu 1998a,b; Zhang \& Mészáros 2001; Gao \& Fan 2006; Lasky et al. 2014).

However, NS rotation also opens up the possibility of further, independent constraints on the EOS. First of all, accurate timing of pulsars in compact binaries over long periods can constrain the spin-orbit coupling and allow for measurements of the moment of inertia of the NS (see e.g. Bejger et al. 2005; Kramer \& Wex 2009; Watts et al. 2015; Patruno et al. 2017b). Another potential probe of NS structure and EOS is the determination of the mass-shedding limit, that is, the maximum rotation rate the star can sustain before mass is stripped from the outer layers. Measurements of spins of millisecond radio pulsars and accreting millisecond X-ray pulsars are then a valuable tool to place lower limits on the maximum rotation rate of an NS.

While theoretical models based on state of the art modelling of the EOS for hadronic matter all predict maximum frequencies well above $1 \mathrm{kHz}$ (see e.g. Haensel et al. 2009), observations in radio and in X-rays, however, have not led to the discovery of any NS rotating with sub-millisecond periods; the fastest known pulsar, PSR J1748-2446ad, rotates at $716 \mathrm{~Hz}$ (Hessels et al. 2006). In particular, a recent analysis of the spin distribution of accreting NSs in low-mass X-ray binaries (LMXBs) by Patruno et al. (2017a) revealed also that the distribution is well described by two populations, a slower one that peaks around $300 \mathrm{~Hz}$ and a much narrower population (standard deviation $\sigma \approx 30 \mathrm{~Hz}$ ) of fast systems above $540 \mathrm{~Hz}$, with an average frequency of $575 \mathrm{~Hz}$. Standard accretion models, even accounting for the transient nature of the spin-up over repeated accretion episodes, struggle to explain the lack of fast pulsars (Patruno et al. 2017a; D'Angelo 2017; Bhattacharyya \& Chakrabarty 2017), and it has thus been suggested that an additional spin-down torque, which grows rapidly for high-frequencies, is required to explain it (although see Patruno et al. 2012 for a discussion of how accretion torques alone can explain the data). Gravitational waves (GWs) provide a natural mechanism, and it has been suggested that the lack of sub-millisecond pulsars is an indication of quadrupolar "mountains" in the crust or of unstable modes in the core, radiating gravitationally at a level sufficient to balance the spin-up torque due to accretion (Papaloizou \& Pringle 1978; Wagoner 1984; Bildsten 1998; Andersson 1998). This scenario is highly uncertain, however, and estimates of GW emission from known systems, based on theoretical models, indicate a much lower level of emission in most cases (Haskell et al. 2015; Haskell \& Patruno 2017), while current GW detectors, such as advanced LIGO and Virgo, are not yet sensitive enough to detect the waves directly (Abbott et al. 2017a).

In this paper we approach the problem from a different angle. Rather than employing a microphysically motivated EOS, we use an extreme EOS making use only of causality. We do not assume any additional spin-down torques that prevent the NSs from spinning up to their breakup frequency, but rather assume that the NSs have indeed spun up to their maximum frequency, the mass-shedding frequency. Our goal is thus to understand whether the lack of rapidly rotating pulsars (above $f \approx 700 \mathrm{~Hz}$ ) can be explained without additional spin-down torques, and determine what this would mean for the physics of the highdensity interior of the star.

To do this, we followed the approach of Koranda et al. (1997) (see also Silva \& Yunes 2018) and first of all established how low the break-up frequency can be by making only basic physical assumptions, that is, imposing that the EOS remain causal in the core of the star. Below the nuclear saturation density, we adopt a realistic crust EOS, as we describe in the following sections. We analysed the impact on the stellar rotation rate by also imposing that the maximum mass of an NS has to be at least the currently observed maximum of $2 M_{\odot}$ and that the mass-shedding frequency must exceed the currently observed maximum rotation rate of $f \approx 716 \mathrm{~Hz}$.

We then considered whether the lack of NS spinning faster than $f \approx 700 \mathrm{~Hz}$ is consistent with causality and our understanding of low-density physics. As our EOS is the stiffest possible, any realistic EOS will give a higher breakup frequency. Additional physics is needed to explain the lack of fast pulsars, either in the form of exterior spin-down torques (e.g. due to GW emission), or in the form of a phase transition in the interior of the star that softens an otherwise stiff hadronic EOS at high densities, and leads to collapse (either to a black hole or a hybrid star) if accretion pushes the stellar mass above $M \approx 2 M_{\odot}$ (Bejger et al. 2017).

\section{Maximum rotation frequency of neutron stars}

The maximum rotation rate of an NS is determined by the frequency $f_{\max }$ of a test particle co-rotating at the star's equator: this is the so-called mass-shedding frequency limit, above which matter is no longer bound and is ejected from the outer layers of the star. The frequency $f_{\max }$ at the equator depends on the gravitational mass of the star and depends on the EOS. The determination of $f_{\max }$ generally requires the calculation of rotating general relativistic equilibrium configurations. However, Haensel et al. (2009) have shown that to a good degree of accuracy, the mass-shedding frequency $f_{\max }$ can be determined by the EOS-independent empirical formula

$f_{\max }(M)=C\left(\frac{M}{M_{\odot}}\right)^{1 / 2}\left(\frac{R}{10 \mathrm{~km}}\right)^{-3 / 2}$,

where $M$ is the gravitational mass of the rotating star, $R$ is the radius of the non-rotating star of mass $M$, and $C$ is a prefactor that is approximately EOS independent and only depends on whether we are dealing with a hadronic (low density at the surface) or a self-bound (strange) star. For standard hadronic matter, the coefficient is $C_{\mathrm{NS}}=1.08 \mathrm{kHz}$, while for self-bound strange stars (with a hadronic outer crust), it is $C_{\mathrm{S}}=1.15 \mathrm{kHz}$, with an accuracy of a few percent, as discussed below (Haensel et al. 2009). The second value of $C$ is also appropriate for any EOS that is self-bound at high density (of the order of nuclear matter density) and thus should also be used for the limiting EOS we construct in the following, the so-called "causal" EOS, that is maximally stiff and corresponds to matter in which the speed of sound is equal to the speed of light. The difference between the two values of $C$ is an example of the role of the outer layers of the NS (the crust), as their response to fast rotation in the equatorial plane is stronger than that of the NS core, thus making a quantitative difference in the case of strange stars with only an outer crust (for a detailed analysis, see Zdunik et al. 2001, where the role of the outer crust on rotating strange stars was discussed). The formula above can be used for masses up to $0.9 M_{\max }^{\text {stat }}$, with $M_{\max }^{\text {stat }}$ the maximum mass of the non-rotating configuration. We note that as we discuss in the following, this precludes us from using it to estimate the absolute maximum mass-shedding frequency for a given EOS, as this will generally correspond to the maximum mass configuration. Nevertheless, Eq. (1) is an important tool for estimating the mass-shedding frequency of the majority of the configurations and the average density in the interior of the NS.

The relative errors when comparing results of Eq. (1) to numerical calculations are typically within $2 \%$, with largest 
Table 1. Mean density (in units of the saturation density $\rho_{0}=2.7 \times$ $10^{14} \mathrm{~g} \mathrm{~cm}^{-3}$ ) and stellar radius (in km) of an NS with $M=2 M_{\odot}$ for the two values of $C$ entering in Eq. (1) and for $f_{\max }$ equal to the largest observed spin frequency $(716 \mathrm{~Hz})$ and to $800 \mathrm{~Hz}$.

\begin{tabular}{cllll}
\hline \hline \multirow{2}{*}{$f_{\max }$} & \multicolumn{2}{c}{$C=1.08 \mathrm{kHz}$} & \multicolumn{2}{c}{$C=1.15 \mathrm{kHz}$} \\
\cline { 2 - 5 } & $\bar{\rho}$ & $R\left(2 M_{\odot}\right)$ & $\bar{\rho}$ & $R\left(2 M_{\odot}\right)$ \\
\hline 716 & 0.77 & 16.56 & 0.68 & 17.28 \\
800 & 0.97 & 15.39 & 0.85 & 16.04 \\
\hline
\end{tabular}

deviations of $5 \%$ at highest masses, although the precision of the formula worsens slightly on the low-mass side for masses below $0.5 M_{\odot}$. A detailed discussion of Eq. (1) for different EOSs and the accuracy of these approximations is presented in Haensel et al. (2009). It can be inverted to obtain the mean density of the non-rotating star for which the mass-shedding frequency is $f_{\max }$ at a given mass,

$\bar{\rho}=1.76 \rho_{0} \frac{f_{\mathrm{max}}^{2}}{C^{2}}$,

which decreases with decreasing maximum frequency. In Table 1 we show the mean densities and radii of maximally rotating configurations with a mass $M=2 M_{\odot}$. It is clear that low maximum frequencies, close to the currently observed maximum of $716 \mathrm{~Hz}$, will lead to very low average densities, below the nuclear saturation density $\rho_{0}$. As we show below, in this range of densities, the EOS for the crust is constrained well enough to limit how stiff the EOS can be and it limits the possibility of having low values for the breakup frequency.

Equation (1) shows that the mass-shedding frequency increases with the mass, and is thus maximum for the maximum mass $M$ that can be obtained for a given EOS. This value depends on the choice of EOS, and is furthermore beyond the range of validity of Eq. (1), which can only be used reliably in the mass range $0.5 M_{\odot}<M<0.9 M_{\max }^{\text {stat }}$. To obtain an absolute upper bound on $f_{\max }$, one can fit maximum mass configurations of NSs that rotate at the breakup frequency, however, and obtain the following expression for the absolute maximum frequency $f_{\max }^{\text {EOS: }}$

$f_{\max }^{\mathrm{EOS}}=C_{\max }\left(\frac{M_{\max }^{\mathrm{stat}}}{M_{\odot}}\right)^{1 / 2}\left(\frac{R_{M_{\max }}^{\mathrm{stat}}}{10 \mathrm{~km}}\right)^{-3 / 2}$,

where $C_{\max }=1.22 \mathrm{kHz}$ (Haensel et al. 1995) and $R_{M_{\max }}^{\text {stat }}$ is the radius of the non-rotating star at the maximum mass $M_{\max }^{\text {stat }}$. $C_{\max }$ is independent of the EOS, while the maximum mass $M_{\max }$ (and corresponding radius) clearly depends on it. We note that close to the mass-shedding frequency, the maximum mass of the rotating star $M_{\max }$ will generally be higher than the maximum mass of the non-rotating configuration $M_{\max }^{\text {stat }}$ by up to $20 \%$ (Lasota et al. 1996). To account for this and estimate the accuracy of the formula in Eq. (3), we have also calculated maximally rigidly rotating models for the EOSs described below with the use of the multi-domain spectral methods library LORENE $^{1}$ (Gourgoulhon et al. 2016) and the nrotstar code (Bonazzola et al. 1993; Gourgoulhon et al. 1999). We also recall the result of Bejger et al. (2007), who demonstrated that with

\footnotetext{
1 http://www. lorene.obspm. fr
}

a great accuracy the mass-shedding frequency may be approximated by

$f_{\max }=\frac{1}{2 \pi} \sqrt{\frac{G M}{R_{\mathrm{eq}}^{3}}}$,

with gravitational mass $M$ and equatorial (circumferential) radius $R_{\text {eq }}$ of a star rotating at the mass-shedding frequency $f_{\max }$. This relation may be used to estimate the maximum radius that a star can support for a given $f_{\max }$ and $M$; for $f_{\max }=716 \mathrm{~Hz}$, we obtain

$R_{\mathrm{eq}}^{\max }=20.94\left(\frac{M}{1.4 M_{\odot}}\right)^{1 / 3} \mathrm{~km}$.

We note that Eqs. (1) and (4) can appear to be two different formulas for the Keplerian frequency. However, this is not the case: we recall that in Eq. (4) the radius $R_{\mathrm{eq}}$ is the equatorial radius of a rotating, oblate configuration, and is $40-50 \%$ larger than the radius $R$ of a non-rotating star of the same gravitational mass (see Fig. 5, Haensel et al. 2009). Eq. (1), on the other hand, allows to determine $f_{\max }$ using the mass and radius $R$ of the nonrotating star, whereas Formula (5) gives the equatorial radius $R_{\text {eq }}$ of the star rotating at the Keplerian frequency.

\section{Causal limit EOS}

Given our ignorance regarding the EOS of matter at supranuclear densities, we did not use a microphysically motivated EOS and only demanded causality throughout the star. As a limiting case, we thus considered the maximally compact causal limit (CL) EOS:

$P=\left(\rho-\rho_{u}\right) c^{2}$,

for which the speed of sound $c_{\mathrm{s}}$ is such that

$c_{\mathrm{s}}^{2}=\frac{\mathrm{d} P}{\mathrm{~d} \rho}=c^{2}$,

where $P$ is the pressure and $\rho_{u}$ is the minimum mass-energy density to which the CL EOS extends, which in our case was a free parameter. For self-bound stars, $\rho_{u}>0$, while for stars with a crust, $\rho_{u}$ is approximately the density at the crust-CL EOS boundary. For the CL EOS the maximum mass $M_{\max }$ of a nonrotating self-bound star is (Glendenning 2000)

$M_{\max }=4.07 \sqrt{\frac{2.7 \times 10^{14} \mathrm{~g} \mathrm{~cm}^{-3}}{\rho_{u}}} M_{\odot}$,

with a radius

$R_{\mathrm{Mmax}}=17.1 \sqrt{\frac{2.7 \times 10^{14} \mathrm{~g} \mathrm{~cm}^{-3}}{\rho_{u}}} \mathrm{~km}$.

We assumed that the highest currently observed spin frequency of $716 \mathrm{~Hz}$ corresponds to the absolute maximum frequency given by Eq. (3). Then using Eq. (2) with $C=1.22 \mathrm{kHz}$, we obtain a mean density of $\bar{\rho}=0.61 \rho_{0}$. As the maximum frequency corresponds to the maximum mass configuration, we can make use of Eqs. (8) and (9) to calculate the mean density of such a star as a function of $\rho_{u}$. We obtain $\rho_{u}=0.43 \rho_{0}$, which is well below the nuclear saturation density. This would thus imply that regions of the star with $\rho<\rho_{0}$ are also described by the CL EOS. This can be excluded from our understanding of low-density NS 


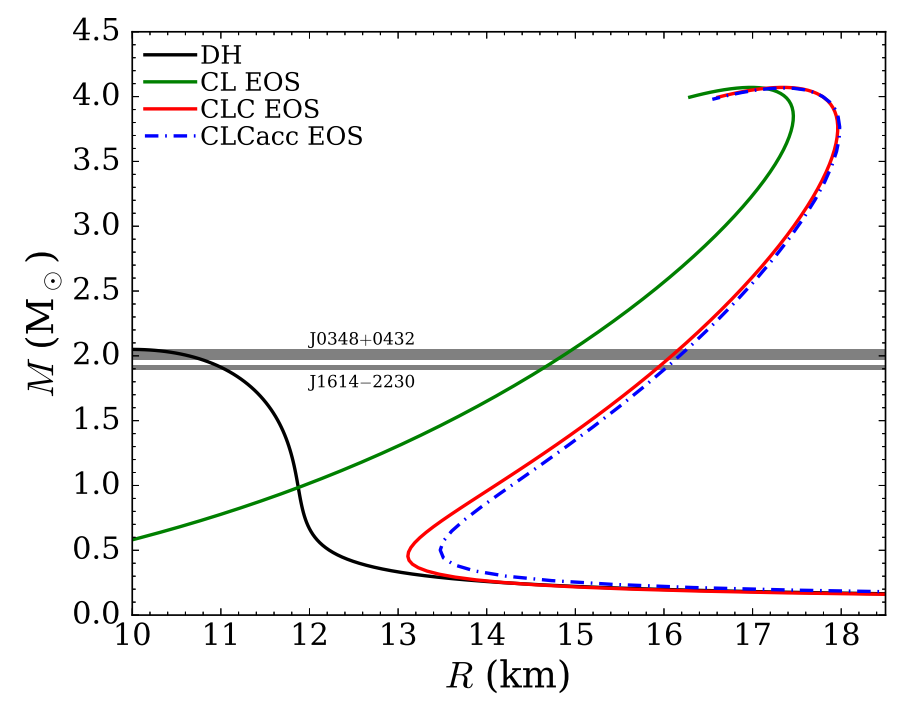

Fig. 1. Mass-radius relations for non-rotating stars for the CL EOS with $\rho_{u}=\rho_{0}$, the CL EOS with two crust models connected at $\rho_{u}=\rho_{0}$ : the catalysed crust from Douchin \& Haensel (2001) (CLC EOS) and the accreted crust from Haensel \& Zdunik (2008) (CLCacc EOS). For reference we plot the $M-R$ relation obtained for the DH EOS. The horizontal lines correspond to the highest observed maximum mass for PSR J1614-2230 and PSR J0348+0432.

physics, however, as despite significant uncertainties regarding composition and transport properties, the stiffness of the crustal EOS is constrained well enough to discard the CL EOS as a viable option in this region (Fortin et al. 2016; Haensel \& Fortin 2017).

If we accept that our ignorance of the EOS begins at densities $\rho>\rho_{0}$, it is thus necessary to construct configurations with $\rho_{u}>\rho_{0}$, and supplement the CL EOS with a realistic crust for lower densities. Before doing this, we note that if in Eq. (8) we take $\rho_{u}=\rho_{0}$, the maximum mass is well above the observed maximum of $\approx 2 M_{\odot}$, and the radius of such a configuration is larger than the range currently suggested by X-ray observations, of between 10 and $14 \mathrm{~km}$ (Özel \& Freire 2016; Haensel et al. 2016).

\section{Including the crust}

To obtain a robust limit of $f_{\max }$, we considered models in which our ignorance is restricted to densities $\rho>\rho_{0}$, that is, to the core of the NS. For the core we used the CL limit EOS $P=\left(\rho-\rho_{u}\right) c^{2}$ described previously, with $\rho_{u}=\rho_{0}\left(1-P_{0} / \rho_{0} c^{2}\right)$ and $P_{0}$ the pressure at the crust-core boundary ${ }^{2}$. For the crust we used a standard EOS from Douchin \& Haensel (2001; DH in the following) for matter below the nuclear matter density $\rho_{0}$ (corresponding to $n_{0}=0.16 \mathrm{fm}^{-3}$ ), and connected it to the core EOS assuming continuity of the chemical potential $\mu$ and pressure $P$ at the crustcore interface, following the approach presented in Zdunik et al. (2017).

The resulting $M(R)$ plots for both the bare CL EOS NS and the CLC EOS (CL EOS with a realistic crust) NS are presented in Fig. 1. The maximum masses for these EOSs are extremely high, as expected for these maximally stiff EOS, much higher than those obtained for a realistic EOS like that from Douchin \& Haensel (2001). The results for the maximum

\footnotetext{
2 Strictly speaking, our construction, which requires continuity of both $P$ and $\rho$, leads to $\rho_{u}$ being smaller than $\rho_{0}$ by approximately $1 \%$.
}

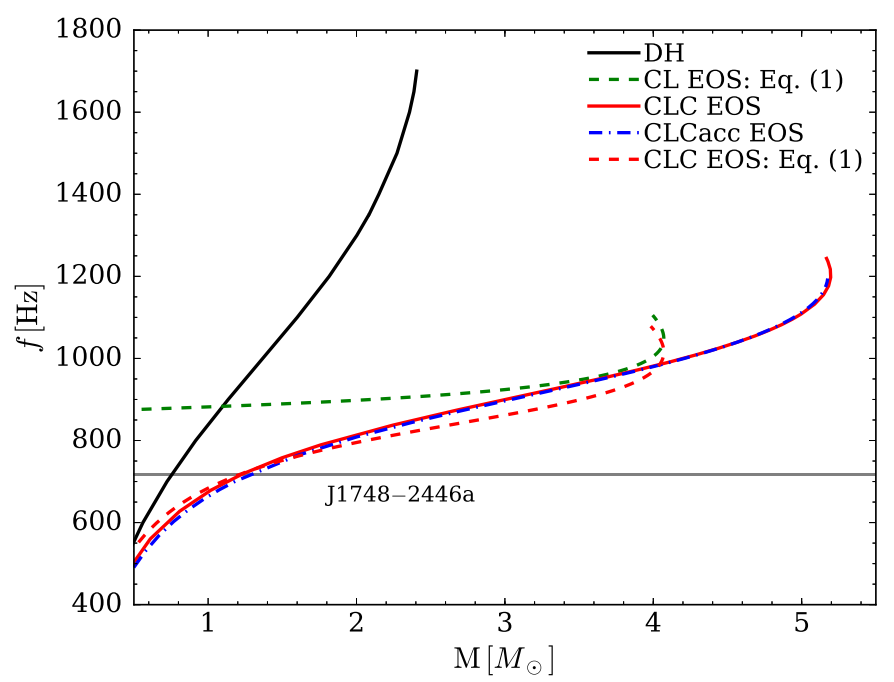

Fig. 2. Mass-shedding frequency vs. mass for the same EOSs as in Fig. 1. For the CLC EOS we plot both the curves obtained with the approximate expression in Eq. (3) from a static configuration and exact numerical solutions for rotating configurations obtained with LORENE. For the DH EOS, configurations also obtained with LORENE are plotted. The maximum mass close to the mass-shedding frequency increases significantly due to the additional rotational support, but the error on the frequency remains small, around $10 \%$. For the most extreme configuration that is physically plausible, the CL EOS with crust, the mass-shedding frequency is around $1200 \mathrm{~Hz}$, well above the observed maximum of $f=716 \mathrm{~Hz}$. As this is the stiffest equation of state that can be built, any softening will lead to a higher mass-shedding frequency, as can be seen by comparing to the curve for the more realistic DH equation of state, which reaches mass shedding around $f \simeq 1730 \mathrm{~Hz}$.

frequency are presented in Fig. 2. The solid lines correspond to calculations obtained using the numerical library LORENE, while the dashed lines use the approximation in Eq. (1). The comparison between the results for the CL EOS with a crust confirms that the approximate formula works well for masses lower than $0.9 M_{\max }^{\text {stat }}$.

It is clear that the crust has a strong effect, as it significantly increases the radius for a given mass compared to a bare CL star, thus decreasing the maximum frequency $f_{k}$. The absolute maximum frequency, corresponding the maximum mass for our CLC EOS (i.e. for $\rho_{u}=\rho_{0}$ ), is $f_{k}^{\max }=1200 \mathrm{~Hz}$. This is the lowest value of the maximum frequency for a hadronic star that is still consistent with our understanding of physics below nuclear saturation density and with minimum physical assumptions for the core, namely that the EOS remain causal. We note that as the CL EOS is the stiffest possible, any realistic EOS such as the DH EOS will lead to a higher value of $f_{k}$ at a given mass. If continued observations of NS spins confirm the absence of NSs spinning at frequencies higher than $f \approx 700 \mathrm{~Hz}$, it can be excluded that this corresponds to $f_{k}^{\max }$, and additional physics is required.

As an example, we show in Fig. $3 M(R)$ for two EOSs, the realistic DH EOS and our CL EOS with crust. We also plot the limit given by Eq. (1) for the currently observed maximum frequency of $f=716 \mathrm{~Hz}$ (dashed lines). The crossing points between this theoretical curve and the $M(R)$ curves give us the minimum mass for which $716 \mathrm{~Hz}$ could correspond to the maximum frequency. It is about $0.8 M_{\odot}$ for $\mathrm{DH}$ model and $1.2 M_{\odot}$ for the CL EOS. The points correspond to pulsars for which there are estimates of both mass and frequency, as given by Haensel et al. (2016) and Özel \& Freire (2016). The uncertainty on the mass determination is marked by the fragment of a curve 


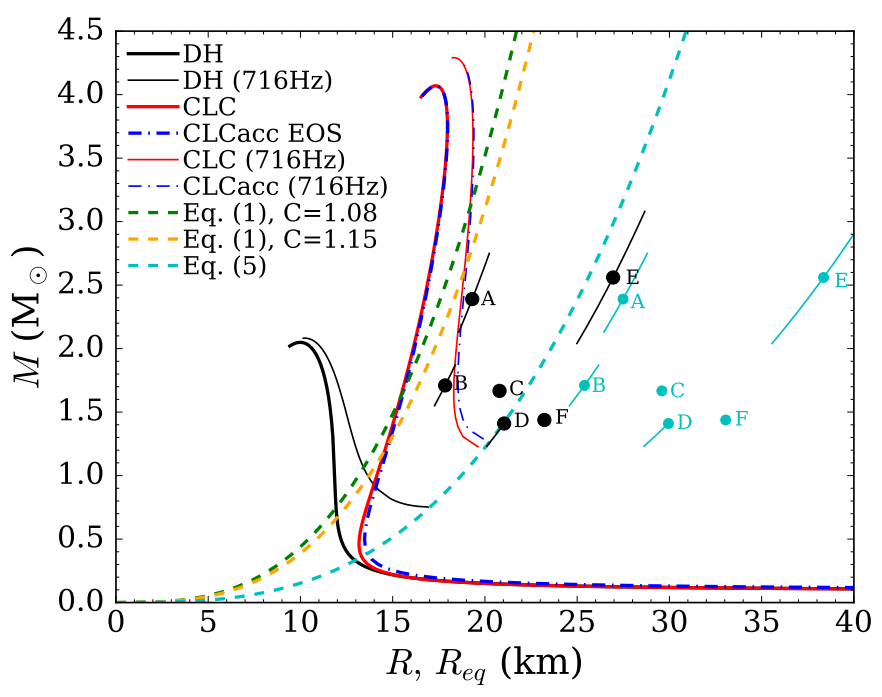

Fig. 3. Mass-radius relations for non-rotating stars for the CL EOS (red) with the crust calculated in Douchin \& Haensel (2001; DH) connected at $\rho_{u}=\rho_{0}$. For reference we plot the $M-R$ relation obtained for the DH EOS. Dashed lines correspond to Eq. (1) for $f=716 \mathrm{~Hz}$ (with two values of the factor $C=1.08$, appropriate for standard hadronic matter, and $C=1.15$, which is used for self-bound stars and is also appropriate for our maximally stiff CL EOS) and Eq. (4) for $f=716 \mathrm{~Hz}$ (the radius is here the equatorial radius of a rotating star). The crossing point between the theoretical $M(R)$ curves and the dashed curves corresponds to the minimum mass for which the mass-shedding frequency of a star described by the chosen EOS could be $f=716 \mathrm{~Hz}$. Black points are observational limits from pulsars for which there are simultaneous frequency measurements and mass determinations, with the error bars determined by the uncertainty on mass, obtained by applying Eq. (1), whereas cyan points correspond to Eq. (4). The letters denote the following rapidly spinning pulsars: A - B1957+20 $(622.12 \mathrm{~Hz}), \mathrm{B}-\mathrm{J} 1023+0038(592.42 \mathrm{~Hz}), \mathrm{C}-$ J1903+0327 (465.14 Hz), D - J2043+1711 (420.19 Hz), E - J1311$3430(390.57 \mathrm{~Hz})$, and $\mathrm{F}-\mathrm{J} 0337+1715(365.95 \mathrm{~Hz})$. Theoretical $M(R)$ relations should be located on the left side of the observed pulsar to be consistent with its parameters. The more constraining points correspond to faster pulsars, so that the detection of objects rotating at frequencies above $\sim 1 \mathrm{kHz}$ would begin to constrain our CL EOS and more generally the physics of dense matter in the stellar interior.

defined by Eq. (1) for the measured frequency of a given object. We see that if NSs rotating at higher frequency were observed, they would begin to provide constraints for models of the highdensity interior.

Our conclusions depend very weakly on the assumptions on the crustal EOS we adopted for the NS (whether it is accreted or catalysed, and which specific model is used). For our analysis the basic parameter of the crust that determines the NS Keplerian frequency is its thickness. The latter depends mainly on the value of the baryon chemical potential $\mu$ at the crust-core interface and at the surface (Zdunik et al. 2017). The detailed description of dense matter in the crust, its composition in particular, influences the microscopic properties such as the heat transport, superfluidity, pinning etc., but not the thickness strongly enough to influence our results. The difference in the radius of a non-rotating NS with an accreted crust and with a catalysed one is $\Delta R(f=0 \mathrm{~Hz}) \sim 100 \mathrm{~m}$ (Zdunik et al. 2017), as is shown in Fig. 1 for the CLCacc model, where the accreted crust from Haensel \& Zdunik (2008) is connected to the CL EOS at $\rho_{0}$. Rotation increases the difference between NSs with accreted and catalysed crusts: $\Delta R(f=500 \mathrm{~Hz}) \sim 120 \mathrm{~m}$ and $\Delta R(f=716 \mathrm{~Hz}) \sim 200 \mathrm{~m}$, which amounts to only $\sim 1 \%$ of the
NS radius, however; see Fig. 3. For example, for Keplerian configurations with $M=2 M_{\odot}$ the frequency for a catalysed crust (corresponding to a more compact NS) is only higher by $\sim 0.6 \%$ and the radius smaller by $\sim 0.4 \%$ compared to a star with an accreted crust; see Fig. 2, where the CLC and CLCacc curves are almost indistinguishable. Our conclusions on the minimum cutoff frequency for accreting NSs are therefore not influenced by current uncertainties on the crust EOS.

\section{Conclusions}

We have examined the problem of whether, given our lack of understanding of high-density physics, the observed limit on the rotation rate of NSs of $f \approx 700 \mathrm{~Hz}$ (Patruno et al. 2017a) can correspond to the maximum rotation frequency of the star, or additional spin-down torques (due e.g. to GW emission or additional spin-down torques in a magnetised accretion disc) must be invoked.

We investigated this in an EOS-independent way by following the approach of Koranda et al. (1997) and making only minimum physical assumptions, namely that our models remain causal in the high-density interior, and that the EOS below nuclear saturation density $\rho_{0}$ is given by the realistic model of Douchin \& Haensel (2001). This produced a maximally stiff EOS that will give the lowest possible breakup frequency for a NS. Any softening (as all realistic models will provide) will lead to a higher maximum frequency.

We find that the maximum rotational frequency for an NS cannot be lower than $f_{\max } \approx 1200 \mathrm{~Hz}$, and that the observed lack of NSs spinning faster than $\approx 700 \mathrm{~Hz}$ is not consistent with minimum physical assumptions on hadronic physics, ruling out centrifugal breakup as the mechanism limiting the spin of the observed systems. Additional mechanisms must be at work to explain this.

The first possibility is most commonly considered: additional spindown torques acting on the NS, either due to GWs or to interactions between the disc and the magnetic field of the star (Andersson 1998; Bildsten 1998; Patruno et al. 2012). Another possibility emerges, however, if we observe Fig. 2. For masses $M>2 M_{\odot}$, the maximum frequency $f_{k} \gtrsim 810 \mathrm{~Hz}$. The currently observed limit for the spin frequency would thus be roughly consistent with our CL EOS+crust if we are not observing stars with $M \gtrsim 2 M_{\odot}$. We recall, however, that this is only the case for our CL core EOS, and that realistic EOSs with maximum mass close to $M_{\max }^{\text {stat }} \approx 2 M_{\odot}$ are generally much softer and predict much higher values of $f_{k}$. It would thus be necessary for the EOS to be very stiff (close to the CL EOS) and thus predict a high maximum mass. Selection effects might be preventing us from observing higher mass NSs. One such possibility might be related to the binary evolution of the LMXB where the recycling process occurs. Tauris et al. (2012) suggested that the equilibrium spin period that an NS can reach during the recycling process is a steep function of the total mass that is accreted. The mass transfer process (from the donor star to the surface of the NS) is almost certainly not conservative, since there is evidence for mass ejection phenomena in LMXBs (e.g. relativistic jets, donor ablation, and accretion disk winds). This in turn reduces the maximum amount of mass available for the spin-up, possibly limiting the maximum spin frequency that NSs can reach. However, we note that in this case, one would expect a smooth decrease in the number of fast NS, and not the existence of a fast population as observed in accreting systems. Furthermore, recent observations by (Linares et al. 2018) suggest a mass $2.3 M_{\odot}$ for a recycled NS. 


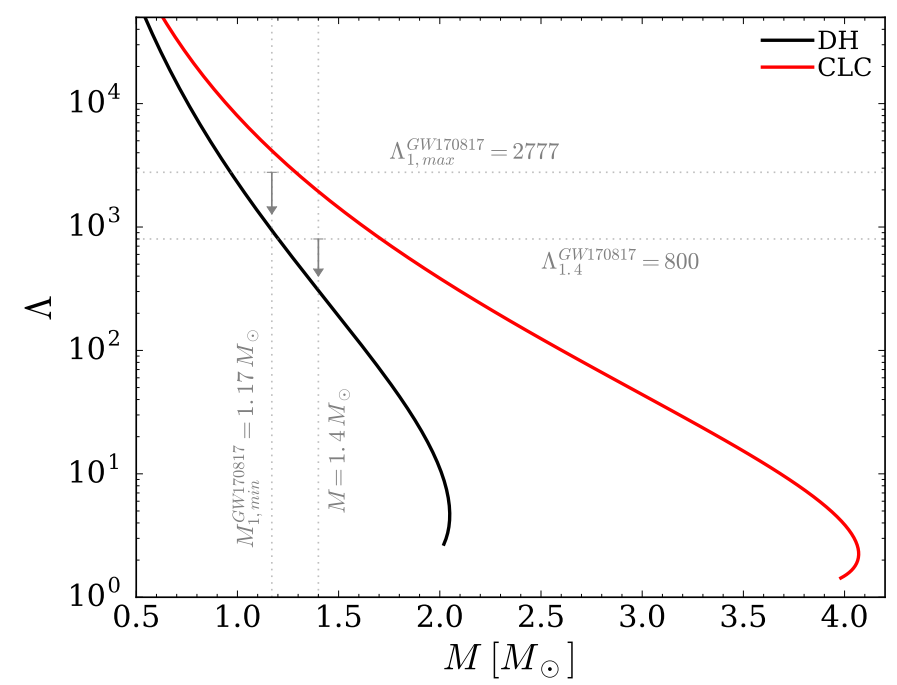

Fig. 4. Tidal deformability $\Lambda$ as a function of mass for the CLC EOS (red) with the DH crust connected at $\rho_{u}=\rho_{0}$ (results obtained with the CLCacc EOS are indistinguishable), and for the DH EOS (green). The bottom horizontal line corresponds to the limit on the deformability of a $1.4 M_{\odot}$ obtained from the observations of GW170817, the merger of two NSs: $\Lambda \leq 800$. While the DH EOS is compatible with this observational constraint, the maximally stiff CLC EOS gives much higher values of $\Lambda$ for $M=1.4 M_{\odot}$. The top horizontal line corresponds to the tidal deformability in the case in which one of the components of the GW170817 merger was a black hole (or a very compact selfbound star) with vanishing deformability. This limiting $\Lambda_{1, \max }=2777$ was computed from the observational bound on $\tilde{\Lambda}=800$ (Eq. (10)) for marginal values of the component masses, $M_{1}=1.17 M_{\odot}$ (assumed NS, $\Lambda \neq 0$ ) and $M_{2}=1.6 M_{\odot}$ (assumed BH, $\Lambda \equiv 0$ ) in the high-spin prior estimation case (see Table 1 in Abbott et al. 2017b for details).

Another intriguing possibility is that the EOS is indeed very stiff, close to the CLC EOS, but there is significant softening at high densities, leading to back-bending in the EOS, which leads to a collapse for masses higher than $M \approx 2 M_{\odot}$, either directly to a black hole, or to a stable branch of hybrid stars (Gerlach 1968; Glendenning \& Kettner 2000; Bejger et al. 2017). We note, however, that if the system collapses to a more compact configuration, we may expect it to be more rapidly rotating if angular momentum is conserved, and such systems are not observed, although the dynamics of such a collapse are poorly understood. Future work should aim to evaluate the viability of this scenario in more detail.

Furthermore, this model would still require the EOS for hadronic matter to be close to the causal limit, much stiffer than what most models predict and in tension with the limits set by the recent measurements of tidal deformability obtained in GWs from the merger of two NSs, event GW170817 (Abbott et al. 2017b; see also Paschalidis et al. 2018, De et al. 2018 for a recent analysis). For this event, one has a constraint $\tilde{\Lambda} \leq 800$ ( $90 \%$ credible interval) for a high NS spin prior, where

$\tilde{\Lambda}=\frac{16}{13} \frac{\left(M_{1}+12 M_{2}\right) M_{1}^{4} \Lambda_{1}+\left(M_{2}+12 M_{1}\right) M_{2}^{4} \Lambda_{2}}{\left(M_{1}+M_{2}\right)^{5}}$,

can be translated into the deformability of a single NS of $1.4 M_{\odot}$, leading to $\Lambda_{1.4} \leq 800$ (Abbott et al. 2017b; The LIGO Scientific Collaboration et al. 2018). We compare this limit with the DH EOS and the CLC EOS in Fig. 4, which shows that the CLC EOS is incompatible with it. Additionally, it might be assumed that one of the components in the GW170817 system was a black hole (or a self-bound star) with a vanishing tidal deformability. In that case, if we assume that $\Lambda_{2} \equiv 0$, for example, then

$$
\Lambda_{1}=\frac{13}{16} \frac{\left(M_{1}+M_{2}\right)^{5}}{\left(M_{1}+12 M_{2}\right) m_{1}^{4}} \tilde{\Lambda} .
$$

To estimate how large $\Lambda_{1}$ is, we can adopt $M_{1}=1.17 M_{\odot}$ and $M_{2}=1.60 M_{\odot}$ (meaning that the NS has mass $M_{1}$ and the black hole mass $M_{2}$ ), and arrive at $\Lambda_{1}<2777$. Even in this extreme case, the CLC EOS is only marginally compatible with this bound. Given the extreme stiffness of our causal EOS, it appears unlikely that the LIGO-Virgo constraint can be reconciled with a $700 \mathrm{~Hz}$ breakup frequency even for low-mass stars.

This further strengthens our conclusion that our lower limit on the Keplerian frequency is robust, as more realistic EOSs will always lead to higher Keplerian frequencies. The observed lack of NSs spinning faster than $\approx 700 \mathrm{~Hz}$ cannot be a consequence to the physical breakup frequency of the NS, and additional physics must be at work in these systems to prevent the stars from spinning up to higher rotation rates.

Acknowledgements. We acknowledge support from the Polish National Science Centre (NCN) via SONATA BIS 2015/18/E/ST9/00577 (BH) and 2016/22/E/ST9/00037 (MB) and from the European Union's Horizon 2020 research and innovation programme under grant agreement No. 702713 and No. 653477. Partial support comes from PHAROS, COST Action CA16214. AP acknowledges support from an NWO (Netherlands Organization for Scientific Research) Vidi Fellowship. JLZ and MF were supported by the Polish National Science Centre (NCN) grant UMO-2014/13/B/ST9/02621.

\section{References}

Abbott, B. P., Abbott, R., Abbott, T. D., et al. 2017a, ApJ, 839, 12

Abbott, B. P., Abbott, R., Abbott, T. D., et al. 2017b, Phys. Rev. Lett., 119, 161101

Andersson, N. 1998, ApJ, 502, 708

Antoniadis, J., Freire, P. C. C., Wex, N., et al. 2013, Science, 340, 448

Arzoumanian, Z., Gendreau, K. C., Baker, C. L., et al. 2014, in Space Telescopes and Instrumentation 2014: Ultraviolet to Gamma Ray, Proc. SPIE, 9144, 914420

Arzoumanian, Z., Brazier, A., Burke-Spolaor, S., et al. 2018, ApJS, 235, 37

Bassa, C. G., Pleunis, Z., Hessels, J. W. T., et al. 2017, ApJ, 846, L20

Bejger, M., Bulik, T., \& Haensel, P. 2005, MNRAS, 364, 635

Bejger, M., Haensel, P., \& Zdunik, J. L. 2007, A\&A, 464, L49

Bejger, M., Blaschke, D., Haensel, P., Zdunik, J. L., \& Fortin, M. 2017, A\&A, 600, A39

Bhattacharyya, S., \& Chakrabarty, D. 2017, ApJ, 835, 4

Bildsten, L. 1998, ApJ, 501, L89

Bonazzola, S., Gourgoulhon, E., Salgado, M., \& Marck, J. A. 1993, A\&A, 278, 421

Dai, Z. G., \& Lu, T. 1998a, A\&A, 333, L87

Dai, Z. G., \& Lu, T. 1998b, Phys. Rev. Lett., 81, 4301

D’Angelo, C. R. 2017, MNRAS, 470, 3316

De, S., Finstad, D., Lattimer, J. M., et al. 2018, Phys. Rev. Lett., 121, 091102

Demorest, P., Pennucci, T., Ransom, S., Roberts, M., \& Hessels, J. 2010, Nature, 467, 1081

Douchin, F., \& Haensel, P. 2001, A\&A, 380, 151

Falcke, H., \& Rezzolla, L. 2014, A\&A, 562, A137

Fonseca, E., Pennucci, T. T., Ellis, J. A., et al. 2016, ApJ, 832, 167

Fortin, M., Providência, C., Raduta, A. R., et al. 2016, Phys. Rev. C, 94, 035804

Gao, W.-H., \& Fan, Y.-Z. 2006, Chin. J. Astron. Astrophys., 6, 513

Gerlach, U. H. 1968, Phys. Rev., 172, 1325

Glendenning, N. K. 2000, Compact Stars: Nuclear Physics, Particle Physics, and General Relativity (New York: Springer), 467

Glendenning, N. K., \& Kettner, C. 2000, A\&A, 353, L9

Gourgoulhon, E., Haensel, P., Livine, R., et al. 1999, A\&A, 349, 851

Gourgoulhon, E., Grandclément, P., Marck, J. A., Novak, J., \& Taniguchi, K.

2016, Astrophysics Source Code Library [record ascl:1608. 018]

Haensel, P., \& Fortin, M. 2017, J. Phys. Conf. Ser., 932, 012034

Haensel, P., Salgado, M., \& Bonazzola, S. 1995, A\&A, 296, 745 
B. Haskell et al.: Fundamental physics and the absence of sub-millisecond pulsars

Haensel, P., Potekhin, A. Y., \& Yakovlev, D. G. 2007, in Neutron Stars 1: Equation of State and Structure, Astrophysics and Space Science Library, 326 Haensel, P., \& Zdunik, J. L. 2008, A\&A, 480, 459

Haensel, P., Zdunik, J. L., Bejger, M., \& Lattimer, J. M. 2009, A\&A, 502, 605

Haensel, P., Bejger, M., Fortin, M., \& Zdunik, L. 2016, Eur. Phys. J. A, 52, 59

Haskell, B., \& Patruno, A. 2017, Phys. Rev. Lett., 119, 161103

Haskell, B., Priymak, M., Patruno, A., et al. 2015, MNRAS, 450, 2393

Hessels, J. W. T., Ransom, S. M., Stairs, I. H., et al. 2006, Science, 311, 1901

Koranda, S., Stergioulas, N., \& Friedman, J. L. 1997, ApJ, 488, 799

Kramer, M., \& Wex, N. 2009, Class. Quant. Grav., 26, 073001

Lasky, P. D., Haskell, B., Ravi, V., Howell, E. J., \& Coward, D. M. 2014, Phys. Rev. D, 89, 047302

Lasota, J.-P., Haensel, P., \& Abramowicz, M. A. 1996, ApJ, 456, 300

Linares, M., Shahbaz, T., \& Casares, J. 2018, ApJ, 859, 54

Motch, C., Wilms, J., Barret, D., et al. 2013, ArXiv e-prints [arXiv:1306.2334]

Özel, F., \& Freire, P. 2016, ARA\&A, 54, 401

Papaloizou, J., \& Pringle, J. E. 1978, MNRAS, 184, 501
Papitto, A., Torres, D. F., Rea, N., \& Tauris, T. M. 2014, A\&A, 566, A64 Paschalidis, V., Yagi, K., Alvarez-Castillo, D., Blaschke, D. B., \& Sedrakian, A. 2018, Phys. Rev. D, 97, 084038

Patruno, A., Haskell, B., \& D'Angelo, C. 2012, ApJ, 746, 9

Patruno, A., Haskell, B., \& Andersson, N. 2017a, ApJ, 850, 106

Patruno, A., Jaodand, A., Kuiper, L., et al. 2017b, ApJ, 841, 98

Ravi, V., \& Lasky, P. D. 2014, MNRAS, 441, 2433

Sieniawska, M., Bejger, M., \& Haskell, B. 2018, A\&A, 616, A105

Silva, H. O., \& Yunes, N. 2018, Classical and Quantum Gravity, 35, 015005

Tauris, T. M., Langer, N., \& Kramer, M. 2012, MNRAS, 425, 1601

The LIGO Scientific Collaboration, the Virgo Collaboration, Abbott, B. P., et al. 2018, ArXiv e-prints [arXiv:1805.11579]

Wagoner, R. V. 1984, ApJ, 278, 345

Watts, A., Espinoza, C. M., Xu, R., et al. 2015, Advancing Astrophysics with the Square Kilometre Array (AASKA14), 43

Zdunik, J. L., Fortin, M., \& Haensel, P. 2017, A\&A, 599, A119

Zdunik, J. L., Haensel, P., \& Gourgoulhon, E. 2001, A\&A, 372, 535

Zhang, B., \& Mészáros, P. 2001, ApJ, 552, L35 